\title{
L'ON FAICT SÇAVOIR QUE L'ON VENDRA. EL ANUNCIO Y CATÁLOGO IMPRESO DE LA SUBASTA DE PINTURAS DEL PRÍNCIPE DE PORTUGAL (BRUSELAS, 1670)
}

\author{
L'ON FAICT SÇAVOIR QUE L'ON VENDRA. THE ADVERTISEMENT \\ AND PRINTED CATALOGUE OF THE AUCTION OF PAINTINGS OWNED \\ BY THE PRINCE OF PORTUGAL (BRUSSELS, 1670)
}

\section{Fernando Bouza}

Universidad Complutense de Madrid

ABSTRACT: In 1670, in Brussels, a printed advertisement announced the auction of a valuable set of a hundred paintings, property of the late Fernando Alejandro de Portugal. A printed broadside announced the sale, and included the names of the artists and titles of the works, as well as their formats and the material form of the pieces, in some cases. Therefore, the advertisement is also an auction catalogue, one of the first to be printed in early modern Europe. This article seeks to tell the story of the print history of the humble broadside as advertisement and catalogue, as well as its content and information on the owners of this extraordinary collection of paintings.

KEYWORDS: Printed Auction Advertisements; Printed Sale Catalogues; Art Collecting; Portugal-Orange Family.

RESUMEN: En 1670 se imprimía en Bruselas el anuncio de la subasta de un centenar de valiosas pinturas que habían sido propiedad de Fernando Alejandro de Portugal. El impreso contenía los nombres de los autores y los títulos de las pinturas, así como, en ocasiones, su soporte y formato. Por ello, el anuncio es también un catálogo de subasta, uno de los primeros que se imprimieron en la Europa altomoderna. El artículo intenta acercarse a la pequeña historia de la impresión del anuncio-catálogo, así como de su contenido y de los propietarios de una extraordinaria colección de pinturas.

PALABRAS CLAVES: Anuncios de subastas impresos; catálogos de venta impresos; coleccionismo artístico; familia Portugal-Orange. 
En la historia del coleccionismo europeo del siglo xvi destaca por méritos propios la figura de Diego (Jacob/Diogo) Duarte, de Amberes (Paepe, 2010; 2017). ${ }^{1}$ Con justicia, este mercader de origen lusosefardí se ha hecho célebre porque fue uno de los muy escasos clientes conocidos que Jan Vermeer tuvo en vida (Montias, 1989: 257). Su colección de pinturas era extraordinaria y está por fortuna bien documentada (Denucé, 1932: 345; Speth-Holterhoff, 1957: 23-24; Dogaer, 1971; Samuel, 1976). Se sabe, por ejemplo, que en 1670 Diego Duarte se hacía, en Amberes, con una pintura de Rafael de Urbino «representante nostre Dame, nostre Seigneur et S. Jean" que antes había sido de Manuel Eugenio de Portugal, el último descendiente de D. Antonio, el Prior do Crato que pretendió la corona lusitana como hijo y heredero del infante D. Luís de Avís.

En 1949, el historiador del arte Jan Denucé publicó el escrito de entrega del cuadro firmado por D. Manuel en Amberes un 23 de marzo de 1670, consignando de dónde provenía la pintura y cómo había llegado a sus manos. ${ }^{2}$ Ese mismo día, el propio Duarte hizo una declaración, inédita hasta ahora, por la que exponía los detalles de la transacción, que no presentaba como una vulgar venta, sino como un trueque. ${ }^{3}$ En ella, explicaba las razones por las que le había pedido a Manuel Eugenio de Portugal que registrase por escrito la procedencia del cuadro, "pour la seule reputation et pour donner du credit a la ditte peinture»"

Se trata de un episodio de indudable importancia para la historia del coleccionismo artístico, porque testimonia que, en una época en la que abundaban las copias y las falsificaciones, reconstruir la procedencia de una pintura era ya un criterio para avalar su autenticidad en el mercado del arte. Así, Diego Duarte anotó que D. Manuel lo había recibido de su hermano Fernando Alejandro - a quien califica como «le grand curieux»-y éste, a su vez, del padre de ambos, Luis Guillermo de Portugal, quien lo obtuvo de su suegro Giovanni Battista Capece, Marqués de Monteleone y tesorero general de Nápoles. ${ }^{5}$ Su rafael, en suma, merecía crédito porque procedía de la reputada colección de los Príncipes de Portugal.

Junto a esta transacción reservadamente privada, testimoniada por sendos manuscritos personales y dotados de cierta solemnidad, otras piezas de la colección pictórica de Fernando Alejandro de Portugal, que había fallecido en Madrid el 24 de diciembre de 1668,

1. Este trabajo se enmarca en el proyecto de investigación MINECO HAR2014-54492-P del Gobierno de España y se ha beneficiado de su financiación. Deseo agradecer a la Dra. María López-Fanjul y Díez del Corral, de los Staatliche Museen zu Berlin, su generosa colaboración en la escritura de este texto.

2. «Monsieur. Je vous envuoye la Peinture de Raphael d’Urbino representante nostre Dame, nostre Seigneur et S. Jean, laquelle est venue du Cabinet de feu Monsieur le Prince mon gran Pere qui l'a donné entre autres pieces en mariage a feu Monsieur le Princé mon Pere, laquelle après sa mort m'est parvenue en mes mains. Je vous souhaite le bonheur, et me dis Monsieur Votre très affectné Serviteur Don Emanuel de Portugal (Anvers le 23 Mars 1670)» [ $N^{\circ} 431$ Emanuel van Portugal aan Duarte (23 Maart 1670)] (Denucé, 1949: 371-372).

3. Conviene advertir que no se trataba de un regalo, tan importantes en la práctica cultural de la alta Edad Moderna, sino de un intercambio por diamantes valorados en 2.200 gulden (Denucé, 1949: lxxv).

4. «Je souligné declare par cette que aujordhuy j’ay troecqué avec son Exce. monseigneur le Prince Don Manuelle de Portugal une peinture representante notre Dame avec notre Seigneur et Saint Jean sur quoy son Excel. m’́a donné un escrit par le quel il est dit que la dette peinture soit de la main de Rafaele Urbino que neantmoins se n'entend point que son dit Exce me la maintendra pour du dit Rafaele d’Urbino, mais que je luy ay prié d'y mettre cette particularité pour la seule reputation et pour donner du credit a la ditte peinture, et non pour obligar son Exce. a chose quoique soit estant un pure troecque. Faict en Anvers ce 23 mars 1670. Diego Duarte», Arquivo Nacional da Torre do Tombo, Lisboa [ANTT], Arquivos de D. António e seus descendentes [ADAD], Caixa 22, Maço 37, Doc. 3512.

5. «Son gran Pere a esté le Prince de Monterian [id est Monteleone] de Naples, et son Pere Dom Loyis de Portugal qui a espousé la Princesse de Monterian, fille du dict Prince. Depuis la mort de son Pere, la peinture est venue a son frere le grand curieux, mort en l'an 1669 et de la a luy» (Denucé, 1949: 372). 
también cambiaron de propietario aquel mismo mes de marzo de $1670 .{ }^{6}$ En este caso, se trató de una venta en subasta pública ${ }^{7}$ para la que, obviamente, se buscó el mejor medio de publicitación que garantizase que «l'on fait sçavoir que l'on vendra».

La historia de la venta de bienes mediante subasta se remonta a la antigüedad clásica y estaba plenamente establecida en el siglo xviI, como ha mostrado John M. Montias en su Art at auction in seventeenth-century Amsterdam, donde señala que Venecia, Amberes y la ciudad holandesa fueron focos de especial importancia para su desarrollo en, respectivamente, los siglos Xv, xVI y xVII (2002). Montias, además, expone que las subastas de obras de arte guardaron en su origen especial relación con las bancarrotas, el pago de deudas y con las testamentarías o casas mortuorias en toda la Europa occidental. ${ }^{8}$

Es cierto que hay que esperar al xviII para que se instauren las grandes casas de subastas permanentes, pero, como se sabe, buena parte de la historia del coleccionismo pictórico de la Edad Moderna se fundamenta en los inventarios de lo que en España se llamaba almonedas de difuntos que, convenientemente pregonadas, se abrían a pujas de particulares. Salvo que pinturas o tapices, pero también armerías, monetarios o bibliotecas, fuesen amortizados mediante un vínculo que impedía su venta testamentaria y garantizaba su preservación mediante transmisión hereditaria, lo más común era que la mayoría de las colecciones se dispersasen en el momento de la muerte de sus propietarios. ${ }^{9}$

En el caso de ciento veinte pinturas que habían sido de Fernando Alejandro de Portugal, el 3 de marzo dio comienzo en Bruselas su pública subasta que se prolongó durante dos días más en una sala de la residencia de Carlo Francesco Airoldi, internuncio apostólico en los Países Bajos españoles. El promotor de la subasta era Pierre Ignace Vendeville, quien declara que había recibido aquellas «cent et vingt pieces de pintures tant grands que petits» del propio Príncipe de Portugal, según consta textualmente en su Estat de ce qui son Exc. Monseigneur le Prince Dom Ferdinand Alexandre de Portugal m'a-t-laissé. ${ }^{10}$

Vendeville era caballerizo - «escuier»- del difunto Príncipe, pero aquellas pinturas no le habían correspondido como fruto de un generoso legado testamentario. Por el contrario, disponía de ellas como garantía del préstamo que le había hecho a su señor con el fin de que pudiese viajar -de hecho, regresar-a la corte madrileña en busca de la gracia real en $1668 .{ }^{11}$

Con la diligencia minuciosa de quien busca reembolsarse una deuda, el acreedor Pierre Ignace Vendeville fue anotando todos los gastos que de su propio peculio había hecho con motivo de la testamentaría de D. Fernando Alejandro desde el 21 de febrero de 1670. Entre ellos, estaba el asiento de haber buscado a unos mozos «pour avoir transporté tous les pintures

6. A la muerte de Fernando Alejandro de Portugal en Madrid, una venta almoneda de las pinturas que había llevado a la corte o reunido allí. La tasación fue realizada por Antonio de Pereda el 9 de abril de 1669 y, entre otras obras, se vendieron dos "banbochos» originales de David Teniers [ "Esternis»], un frutero y "un quadro aovado con su marco de escoltura dorado representando a nuestra señora con el niño Jesús, San Joseph y San Juannico» vendido «en cinqüenta ducados» a Fernando Vergara. ANTT, ADAD, Caixa 24, Maço 40, Doc. 3672.

7. Dio noticia de ella Galesloot, 1868, a partir de una «liste que j’en trouvée par hasard, en classant des archives de l'ancien conseil souverain de Brabant» (Galesloot, 1868: 414). Nosotros trabajamos con otra copia, ANTT, ADAD, Caixa 24, Maço 40, Doc. 3692 [Vente publicque des peintures de la maison Mortuaire de feu le seigneur Prince Don Ferdinand Alexandre de Portugal].

8. Como ejemplo único de una práctica bien conocida, recuérdense las ventas -y compras- de los cuadros de Carlos I de Inglaterra (Brotton, 2006).

9. Hay que recordar las aportaciones del inolvidable Víctor Infantes a propósito de los inventarios de bibliotecas, recogidas en Infantes, 2006.

10. ANTT, ADAD, Caixa 24, Maço 40, Doc. 3677.

11. Este fue el año del reconocimiento definitivo de la entronización de los Braganza en Portugal, lo que, como veremos infra. ponía fin a algunas aspiraciones de los descendientes de D. Antonio.

IMAGO, NÚM. 9, 2017, 39-54 
chez l'Intrernonce», pero también el de pagar "un imprimeur qui a imprimé 400 billets pour la vente des dites pintures». ${ }^{12}$

El tal impresor no era otro que Gielis, o Gilles, Stryckwant I, librero e impresor activo en Lovaina y en Bruselas, donde se presenta en ocasiones como "ordinaris Drukker van de Heeren Staten van Brabant» o "imprimeur ordinaire des Estats de Brabant» (Willems, 1935:42). Así se hace llamar en el pie de imprenta del único ejemplar conservado ${ }^{13}$ de aquella tirada de cuatrocientas copias que Vendeville había hecho imprimir a su costa, es decir:

L'on faict scavoir; Que l'on vendra publicquement au plus haut offrant les Pintures cy suivant, appertenant à la Maison Mortuaire de Feu le Seigneur Prince de Portugal, le 3. de Mars 1670, à la Maison de Monseigneur l'Internonce Apostolicq., en la ville de Bruxelles. A Bruxelles: Chez Gilles Stryckwant, Imprimeur ordinaire des Estats de Brabant, $1670 .{ }^{14}$

Se trataba de un anuncio en un pliego doble impreso por una sola cara en el que se da a conocer la celebración de la venta y el método de subasta, indicando dónde y cuándo comenzaría, así como qué se venderían pinturas. ${ }^{15}$ Sin embargo, la inclusión de una lista en dos columnas con los noventa y nueve asientos para ciento veinte obras en los que se describe, aunque sucintamente, títulos y autores y, en ocasiones, formato y soporte hace que pueda considerarse también un catálogo.

Esta circunstancia lo acercaría a los bien conocidos inventarios impresos de bibliotecas y galerías de pinturas de la época, así como a los abundantes catálogos de librería (Rueda y Agustí, 2016; Infantes y Martínez, 2016). Pero no tanto al modelo que, en la experiencia hispánica, identificamos con el Museo de Montealegre (Madrid: 1677) o la Bibliotheca de Sora (Zaragoza: 1618), sino con índices hechos con el fin de proceder a la venta de una librería particular, ${ }^{16}$ como el Inventario de la de Ramírez de Prado ${ }^{17}$ (Madrid: 1661), el Catálogo general que Claude Bourgeat hizo de la de Arce y Reinoso (Madrid: 1666) o el, menos conocido, Catalogus de la subasta de los libros del pagador general de Flandes Tomás López de Ulloa efectuada en Bruselas en abril de 1659 y de la que se ocupó el librero e impresor Jan Mommaert. ${ }^{18}$

En alguna ocasión, las subastas de una biblioteca podían incluir la venta de algunas pinturas, como sucede en la de Jacques Lescot, Obispo de Chartres, realizada en 1657. Al final del impreso que recoge el catálogo de la venta, se señala "Il y a plusieurs beaux Tableaux, des Trauaux d'Hercule faicts par Francflore [Franz Floris] excellent Peintre, de six pieds d'hault sur quatre pieds de large». ${ }^{19}$

12. ANTT, ADAD, Caixa 24, Maço 40, Doc. 3677 [Pretensions du Sr Pierre Ignace Vendeville, escuier du feu Seigneur Prince Don Alexandre de Portugal contre la maison mortuaire du dit Seigneur Prince de Portugal].

13. No en Short Title Catalogus Vlaanderen, accesible en stcv.be.

14. ANTT, ADAD, Caixa 24, Maço 40, Doc. 3678.

15. Sobre los anuncios impresos, González Sarasa, 2016.

16. Nunca se hubiera debido olvidar la dimensión mercantil del público lector, como bien ilustra el reciente Clavería, 2017.

17. Esteban de Aguilar y Zúñiga realizó el inventario con el objetivo de que la biblioteca fuera «vendue par classes et dispersée» (Gutiérrez, 1977: 195).

18. Catalogus ... Librorum ... D. Thomae Lopez de Vlloa ... Quorum Auctio habebitur Bruxellis die tertiâ, $\theta$ segg. mensis Martii, MDCLIX. [Bruxellis]: Directore Ioanne Mommartio, Bibliopola \& Typographo jurato, sub Insigni Typographiae, [1659]. Por su importancia para las colecciones de libros del Siglo de Oro, se debe destacar la subasta de Cornelius Lerke, Catalogus selectissimorum librorum... Cornelii Lerke, quondam Regis Daniae ad Hispanos Legati... quorum auctio instituenda est Hafniae... ad diem [18] septembris anno 1682, Hafniae: Literis Conradi Hartvigi Neuhofii [1682].

19. Bibliotheca illustrissimi et reverendissimi in Christo Patris D. D. Jacobi Lescot, Carnot. Episcopi, S.1.: n.i., n.a. [1657], 28. 
De hecho, algunos de los primeros subasteros de libros también lo fueron de pinturas. Éste es el caso, por ejemplo, del "early auctioneer» Edward Millington ${ }^{20}$ que se ocupó de la venta de librero oxoniense Richard Davis en 1689, pero también de una Auctio Davisiana de cien pinturas en 1691. ${ }^{21}$ Ese mismo año, apareció la Auctio Millingtoniana Picturarum Omnigenarum, e c:, un catálogo impreso en el que se deja constancia de que se trataba de «A Collection of Extraordinary Paintings \& Limnings, with Variety of the Finest Italian, French, and Other Prints, (several of Them Originals) by the Best Masters. With Many Curiosities of the China Make, and Other Artificial Rarities».22

En suma, la estrecha relación entre subastas de libros y de pinturas hace que el primer catálogo impreso conocido que publicita la venta de cuadros por pujas parezca, de hecho, uno de los habituales inventarios de librería en cuarto. Se trata del Catalogue des raretez de Peeter Stevens, limosnero de Amberes, publicado en 1668 por el impresor amberino Marcelis Parys. ${ }^{23}$

La recetería bruselense de Gielis Stryckwant no es muy pulcra tipográficamente, sin duda porque se imprimió con toda celeridad. El propio Vendeville, recuérdese, señala que la había encargado después del 21 de febrero y hubo de estar impresa con anterioridad al 3 de marzo, fecha de inicio de la venta. Impresa por una sola cara, es posible que fuese pegada en los muros de las calles de Bruselas. A eso apunta, además, otro asiento de gastos de Vendeville, que reza "Item pour avoir fait affaiser (sic) [¿ «afficher»?] les billets trois fois pour toutes les rues». ${ }^{24}$

Sea como fuere, puede ser considerado uno de los primeros ejemplos de catálogo de subasta impreso en el siglo xvII. ${ }^{25}$ No menos relevante es, sin duda y aunque no todos pudiesen ser originales, la nómina de pintores que en él figuran, de Caravaggio y Van Dyck a Holbein y Reni, pero también Rafael, Poussin, Veronés, Guercino, Tintoretto, Tiziano, Correggio, Leonardo, Giorgione, Bellini, Durero, Ribera, El Greco y Velázquez. ${ }^{26}$ Pero ¿quiénes eran estos Príncipes de Portugal que habían llegado a poseer los cuadros que fueron subastados en 1670 ?

Manuel Eugenio y Fernando Alejandro de Portugal eran los únicos hijos de la dama napolitana Anna Capece, cuyo padre era el Marqués de Monteleone, ${ }^{27}$ y de Luis Guillermo

20. Sobre Millington, véase el clásico Lawler, 1898; y, ahora, McElligott, 2005; y Bayer y Page, $2011: 75$.

21. Auctio Davisiana. Picturarum verè originalium: or, A collection of valuable pictures, consisting of the collections, of Cardinal Antonio Barberini. Sir James Palmer, $\theta_{c}$. By Edward Davis, Edward Millington. Catalogues are given at Mr. Sam. Crouch in Cornhil. Richards Coffee-house in Fleetstreet. Mr. Notts in Pall-Mall. Waggoners Coffee-house in the Court of Requests, and at the place of sale, 1691.

22. Will be Sold by Auction, for the Diversion and Entertainment of the Gentlemen, Ladies, \&c. at TunbridgeWells, at the Auction Coffee-house, by the Lower Walks, Near the Wells, on Thursday the 13th of this Instant August, 1691. The Sale Beginning in the Morning at Eight, and Continues Till Twelve in the Forenoon) Except Prayer-time.

23. Catalogue des raretez très renomées de feu Sr. Pierre Stevens en son vivant Aumónier de la ville d'Anvers lesquelles on vendra le 13. du mois d'Aoust $\theta$ jours suivants de cette annee 1668, en la maison mortuaire, situee dans la rue vulgairement nomee la Korte-gasthuys-straet en ladite ville d'Anvers. Antwerp: Marcelis Parijs, [1668]. Lo estudia y publica, SpethHolterhoff, 1957: 14-19; y «Annexe I» 197-199; Briels, 1980.

24. ANTT, ADAD, Caixa 24, Maço 40, Doc. 3677 [Pretensions]. Agradezco a Cécile Vincent-Cassy y a Bernard Vincent su ayuda a la hora de conjeturar sobre el sentido de esta cita.

25. Montias sospechaba que hubieran podido imprimirse algunos catálogos de grandes subastas en la Amsterdam del xviI, en concreto el de la venta Gommer Spranger de 1638, pero señala que "none of these catalogues have survived" (2002: 23).

26. Es importante destacar que los asientos del impreso de Stryckwant incluyen los nombres de los autores de las pinturas, algo que no sucede en la fuente que empleó Galesloot, 1868.

27. Giovanni Battista Capece Galeota, tesorero general de Nápoles, casó con Diana Spinelli

IMAGO, NÚM. 9, 2017, 39-54 
de Portugal. Aunque Manuel era el primogénito, su entrada en el estamento eclesiástico conllevó que renunciase en su hermano pequeño los derechos sucesorios a títulos y rentas cuando su padre falleció en Madrid en 1660. Por línea paterna descendían del Prior do Crato y de Guillermo de Orange, pues Emilia de Nassau, hija del Taciturno y de Ana de Sajonia, se había casado en 1597 con Manuel de Portugal, hijo y heredero de D. Antonio de Portugal (Faria, 1917; Kamp, 1980). Por ello, los Príncipes eran, propiamente, Portugal y NassauSajonia, de forma que no se equivocaba el papa Alejandro VII Chigi cuando se refirió a ellos, en 1660, diciendo "questi signori sono mezzi olandesi». ${ }^{28}$

No obstante, siempre quisieron ser llamados Príncipes de Portugal, manteniendo vivas sus aspiraciones al trono lusitano hasta finales de la década de 1630. Así, como Prinsen van Portugal, son retratados Luis Guillermo y sus dos hijos formando parte del cortejo funeral de las exequias del estatúder Federico Enrique de Orange-Nassau celebradas en 1647.29

Como se sabe, Antonio Prior do Crato rivalizó con Felipe II y otros candidatos en la polémica sucesoria que la desaparición de Sebastián I en Alcazarquivir abrió en 1578. Autoproclamado en 1581 nuevo rey de Portugal, como D. Antonio I, una dura guerra de tres años fue necesaria para derrotar militarmente su pretensión de que él y no Felipe II era el legítimo monarca de Portugal. Hasta su muerte en 1595, el Prior mantuvo su reclamación de la corona y, sin duda, siempre supuso una amenaza importante para el dominio de Felipe II en Portugal, bien porque sus partidarios promovieran revueltas, como las de 1592, bien porque el propio pretendiente desembarcara en 1589 con una armada en las costas portuguesas (Hermann, 2010; Dubert, 2002; Bouza, 2015; Serrão, 1956).

En su continua busca de alianzas contra el poder de Felipe II, D. Antonio desarrolló una ambiciosa política de negociaciones internacionales que lo llevó a mantener relaciones con las cortes de Francia, Inglaterra y Holanda, pero también con la Sublime Puerta, Persia y Marruecos. Incluso, llegó a enviar al Norte de África a uno de sus hijos, D. Cristóvão, quien residió tres años en la corte de Marrakech, mientras que el otro, D. Manuel, lo acompañaba a él en Francia e Inglaterra.

A la muerte de su padre, los Príncipes de Portugal Manuel y Cristóvão escribieron en 1597 una larga carta a la reina Isabel I en la que le presentaban sus deseos de abandonar Inglaterra "et cercher (sic) fortune ailleurs» (Castries, 1906, 31) sin renunciar a luchar contra Felipe II incluso pasando a residir al Norte de África. Pero «avant que prendre celle [voie] d'Affrique (laquelle nous pourons garder [...] pour le dernier refuge)", habían decidido viajar a los Países Bajos, cuyos Estados Generales «ont un ennemy commun avec nous et resistent de tout leur pouvoir à la tirannie exorbitante d'iceluy" (Castries, 1906: 40).

La posibilidad de que los Príncipes portugueses se instalasen en Marruecos junto al sultán Áhmed Al-Mansur parece haber sido bastante real. No obstante, la buena acogida que les prestaron los Estados Generales de las Provincias Unidas y el estatúder Mauricio de Nassau no hizo necesario que los Portugal se instalasen en Marruecos.

Tras vencer numerosos obstáculos confesionales, el matrimonio del pretendiente D. Manuel con Emilia de Nassau-Sajonia, hermana de Mauricio de Nassau, supuso su definitiva incorporación a la corte neerlandesa, abriendo la línea hereditaria de los Portugal-Orange

28. Memoriale [Vida de Manuel Eugenio de Portugal, 1655-1668], ANTT, ADAD, Caixa 42, Maço 98, Doc. 6872. Los apellidos recogidos en el expediente para su ingreso en la orden de Calatrava, de 1656, son Portugal Capece Galeota Nassau y Spinelli, Archivo Histórico Nacional, Madrid, [AHN], Órdenes militares, Caballeros, Calatrava, expediente 2097

29. Se les distingue perfectamente en Pieter Post, Begraeffenisse van syne hoogheyt Frederick Hendrick..., t’Amsterdam: Gedruckt by Nicolaes van Ravensteyn, 1651. 
(Sousa, 1737: 398-399; Piot, 1877). De la unión nacieron numerosos hijos que fueron educados con sus padres en Holanda como miembros de la casa de Orange. Así, el heredero Luis Guillermo de Portugal se crió con su tío Justino de Nassau en la villa de Breda, donde resistió el asedio de las tropas españolas. Cuando la unión terminó en divorcio, ${ }^{30}$ la Princesa Emilia se trasladó con sus hijas a Ginebra, donde está enterrada en la Capilla de Portugal de la catedral calvinista de Saint Pierre (Deonna, 1929:260-262). Por su parte, D. Manuel y D. Luis Guillermo abandonaron Holanda y se dirigieron hacia los Países Bajos meridionales para entrar en la órbita de Isabel Clara Eugenia de Austria y, más tarde, del Cardenal Infante D. Fernando. ${ }^{31}$

Manuel, Príncipe de Portugal, residiría en los Países Bajos reales hasta su muerte en 1638, manteniendo en Bruselas un interesante círculo donde entraron tanto científicos como eruditos. Ya en Holanda, cuando vivía en la corte de los Nassau, había dado pruebas de su curiosidad y, por ejemplo, él y su cuñado, Mauricio de Nassau, estuvieron detrás de la difusión en Europa de la Apología contra los artículos de la ley cristiana de Muhammad Alguazir. Al parecer, durante un banquete en la corte, Manuel y Mauricio le pidieron a Ahmad ibn Abdallah, embajador del Sultán de Marruecos, que les enviase una exposición sobre las razones por las que el Islam debía ser preferido al Cristianismo. A la vuelta a Marruecos, el embajador les envío la Apología que, traducida al latín, circularía más tarde como Epistola theologica de articulis quibusdam fidei ad serenissimos Auriacum et Portugalliae principes scripta (Wiegers, 1996).

Entre los savants que frecuentaron a D. Manuel en estos años de Bruselas se encontraban Jan Baptista van Helmont, el jesuita Andrés Luis de Zamora o al célebre Juan Caramuel. Si el primero propuso en la pequeña academia del Príncipe de Portugal su disertación sobre el "ars volandi», ${ }^{32}$ Zamora y Caramuel colaboraron en la edición del Philippus Prudens Lusitaniae Legitimus Rex Demonstratus, la historia de la sucesión portuguesa de 1580 publicada en Amberes en 1639 que se abre con la extraordinaria constelación astronómica grabada por Erasmus Quellinus. ${ }^{33}$

Escrita en el monasterio cisterciense de San Salvador de Amberes, Caramuel refiere como el Príncipe le había franqueado el acceso a su archivo, donde se encontraban las argumentaciones de los distintos candidatos a suceder a los Avís en 1578-1580. Asimismo, el libro contiene una carta en la que se le ruega al cisterciense que publique el libro para que quede constancia de que los Príncipes reconocen la legitimidad de los Habsburgo como reyes de Portugal. ${ }^{34}$

Para entonces, las pretensiones de los herederos de D. Antonio al trono portugués parecían haber dejado de tener sentido. Hugo Grotius, quien conocía a los Príncipes, los retrata como una especie de pedigüeños que sólo pretenden obtener rentas con las que poder mantenerse. En su juicio sobre la situación portuguesa en 1638, tras la revuelta de Évora, Grotius estima que Francia podría sacar evidente provecho de las alteraciones, pero no apo-

30. Más tarde, volvió a casarse con Luisa Osorio. José de Pellicer, Sucessión de los Reynos de Portugal y el Algarbe, Logroño: por Pedro de Mongastón, 1641, sin foliar.

31. Hay un eco de ellos en los nombres de Manuel Eugenio y Fernando Alejandro de Portugal.

32. Georg Pasch, De novis inventis, Lipsii: Sumptibus Haeredum Joh. Grossi, 1700, 637.

33. Antuerpiae: ex officina Plantiniana Balthasaris Moreti, 1639. Zamora es el «inventor» del tema del frontispicio.

34. Caramuel, Philippus Prudens [Binche, 4 de octubre de 1637] en "Occasio scribendi», preliminares sin foliar. El nombre del fraile figura expresamente en el testamento de D. Manuel, otorgado en Bruselas, 12 de febrero de 1638, ANTT, ADAD, Caixa 8, Maço 12. Por otra parte, la familiaridad era tan grande que el propio Caramuel le pedía a su padre, Lorenzo, que le escribiese "con sobre escrito a Bruselas diciendo en casa del Príncipe de Portugal porque así me inbiará a Lovayna o a donde yo estuviere las cartas», Lovaina, 1 de enero de 1636, AHN, Estado, libro 836, 45. Sobre Caramuel y los Portugal, véase Fernández-Santos 2014: 79.

IMAGO, NÚM. 9, 2017, 39-54 
yando a los Portugal-Nassau, sino a un miembro de la gran nobleza lusitana, bien un Aveiro, bien un Braganza, los únicos personajes que podrían suponer una amenaza verdadera para el poder de los Habsburgo en Portugal. ${ }^{35}$

La pujanza de la nueva realeza de D. Juan IV de Braganza modificó, sin duda, su relación con la corte de los Habsburgo españoles. Madrid siempre se había mostrado reacio a llamarlos Príncipes de Portugal, pero a partir de 1640 aceptaron de hecho esa denominación porque podía significar un detrimento de la legitimidad de los Braganza. Del mismo modo, a cambio del mantenimiento de su fidelidad a los Felipes, la corte española pareció dispuesta a conceder títulos, como el de Trancoso o Sendim, y permitir que se llamasen Duques de Beja. ${ }^{36}$

Estos Príncipes sin patria ni hacienda desarrollaron numerosas estrategias para conseguir mantenerse económicamente. A la postre, además de las rentas holandesas, vivieron de ayudas consignadas sobre rentas en productos de consumo, como el jabón o la nieve. Pero siempre desearon que se les concediesen rentas eclesiásticas que, como los beneficios procurados por Manuel Eugenio, eran concedidas a perpetuidad. Esto hizo que dirigiesen su atención tanto hacia Roma como hacia Madrid, puesto que el monarca católico disponía de jurisdicción beneficial gracias al regio patronato. No obstante, buscaron también relacionarse con banqueros y hombres de negocios, como los cristianos nuevos Castro Ferro (Hierro), los Fonseca o los Cortizos.

Del mismo modo, los Portugal siempre sacaron un gran partido a su colección de pinturas, hecha entre España, Italia y los Países Bajos. Por ejemplo, en 1670, Manuel Eugenio encargó que se presentasen a su parienta Claire Marie de Nassau-Siegen, Princesa de Ligne, cuando llegase a Roma "dos quadros de flores de Bodesson", es decir Nicolas Baudesson. ${ }^{37}$ Cuatro años antes, preparando su partida desde Bruselas rumbo a Madrid, Fernando Alejandro envió una carta a la corte al poderoso Gaspar de Bracamonte, Conde de Peñaranda, acompañada de un «retratillo de Jesu», por desgracia sin más indicaciones que permitan su identificación. ${ }^{38}$

Ese mismo año, 1666, el más pequeño de los Príncipes de Portugal y su madre, Anna Capece, enviaron a un agente, el doctor Lorenzo Astrafeli, a la corte de Juan Jorge II de Sajonia con la pretensión de que el elector protegiese a D. Fernando Alejandro. ${ }^{39}$ Con Astrafeli llegaron a Sajonia varios pinturas de Van Dyck, Fyt, Brill, Lievens y «quatre paysages originaux de Pietro Orrente» (Hantzsch, 1902: 272) que fueron destinadas a la Kurfürstlichen Kunstkammer, origen de la Gemäldegalerie de Dresde. ${ }^{40}$

35. Grocio a Sten Bielke, Lutetiae, 13 febrero de 1638 (Meulenbroek, 1973: 79)

36. No obstante, las relaciones con los Braganza nunca llegaron a romperse del todo, mostrando Luis Guillermo de Portugal en ocasiones una ambigüedad notable entre Madrid y Lisboa.

37. Según se desprende de Carta de Francisco Núñez de Castro a Manuel Eugenio de Portugal, Roma, 21 de julio de 1670, ANTT, ADAD, Caixa 7, Maço 11, Doc. 1111.

38. Fernando Alejandro de Portugal a Antonio Rodríguez de Fonseca Pina, Bruselas, 24 de noviembre de 1666, ANTT, ADAD, Caixa 24, Maço 40, Doc. 3672. Peñaranda era, a la sazón, miembro de la Junta de Gobierno de la regencia con Mariana de Austria.

39. Las cartas al elector con motivo de la embajada Astrafeli de 1666 fueron publicadas en Becmann, 1710: 252253. Están fechadas en Bruselas, 17 de enero de 1666, la de Fernando Alejandro, Príncipe de Portugal; y La Haya, 14 de enero de 1666, la de "D. Anna Prin" de Portugal». La relación de la casa electoral con los Portugal era anterior, como puede verse en la carta (Dresde, 15 de diciembre de 1640) que Juan Jorge I de Sajonía (Johann Georgius Saxoniae) envió al Príncipe Luis Guillermo, ANTT. ADAD. Caixa 12, Maço 20, Doc. 2066.

40. Con toda razón, Matthias Weniger ha llamado la atención sobre la importancia de la llegada de los cuadros de Orrente a Dresde para la historia particular del coleccionismo de artistas españoles en el ámbito alemán (Weniger, 2007: 270). Parece que «[...] Frau Mutter des Prinzen aus Portugal» (Hantzsch, 1902: 272) ha de ser identificada como Anna Capece y no con Luisa de Guzmán. 
Esta práctica del regalo de pinturas la habían debido aprender bien de su padre, Luis Guillermo de Portugal, que también fue generoso con los cuadros de su galería. Así, en 1648, hizo llegar algunos cuadros a manos de Wolfgang Wilhelm, Conde Palatino-Neuburg. Desde Düsseldorf, el Conde Palatino, afamado mecenas de Rubens y coleccionista, le agradecía que «havendo Io recevuto [...] tre pitture mandatemi da V.Eccza cio e una Madona col figliuolo Giesu, l'altra d’una Magdalena et la terza delle Sante Catherina et Margarita, vengo con questa a ringrantiarle affetuosamente Vra. Eccza di quella sua cortesía offrendomi di riservirla nelle occassione del suo gusto». ${ }^{41}$

Hasta que falleció en Roma en 1689, Manuel Eugenio siguió rodeado de pinturas y de artistas, manteniendo una singular correspondencia con Abraham Brueghel a propósito de algunos encargos cuando el flamenco residía en Nápoles. ${ }^{42}$ No obstante, escasean las noticias concretas de la relación con artistas por parte de otros miembros de la familia PortugalNassau, destacando el pago hecho al caravaggiesco Christiaan van Couwenbergh en 1647 por orden de Luis Guillermo de Portugal. ${ }^{43}$

Por su parte, el anuncio impreso por Gielis Stryckwant en 1670 testimonia una parte de la dispersión de las pinturas de los Portugal, en concreto aquella centena que era subastada para satisfacer la deuda con Vendeville. Éste se quedó con algunos cuadros, entre los que se encontraban obras de Caravaggio [10], Van Dyck [51], El Greco [54], Guido Reni [59] o Holbein [91]. ${ }^{44}$ Otros compradores, como Pompeo Giussani o Adrien Mordack, se hicieron, el primero, con piezas de Rafael [2], Tiziano [35, 82] o Dosso Dossi [58] y Tintoretto [67], el segundo. Por último, el estudio de los inventarios de Diego Duarte permitiría conjeturar que el mercader también se hizo con algunas pinturas de los Portugal en la subasta de 1670. De este modo, podría haber adquirido unas perspectivas de Viviano Codazzi [66], un paisaje de Jacques Fouquiéres [19] o una cesta de frutas de Caravaggio [25]. ${ }^{45}$

También habría pasado a manos de Duarte el cuadro del vasariano Lorenzo Sabattini (Lorenzino da Bologna o Laurentius Bononiensis) que es asentado en el número 68 como «La naisssance de Venus de Laurençio Bonomi». El 4 de marzo de 1670, el segundo día de subasta, fue comprado por Pompeo Giussani, pero terminó en manos de Duarte como se dice en la entrada "Een groot stuck de geboorte Venus met veele naeckte figuren vanden Prince van Portugal gehandelt tegen gesteent weert van Lorenso Bonnon guld. 200» de su inventario de 1683 (Dogaer, 1971: n 40). A la postre, el cuadro ha acabado en Pembroke-Wilton House, donde, en 1764, es descrito como "[At the ceiling] Venus's Birth: She is rising out of the Sea, the three Graces attending her: There are also five Cupids in differents Actions. 1.By Lorenzino da Bologna» (Kennedy, 1764:121). ${ }^{46}$

41. 9 de octubre de 1648, ANTT. ADAD, Caixa 12, Maço 20, Doc. 2071.

42. Se trata de nueve cartas datadas en 1685 cuya edición se prepara en estos momentos.

43. Orden de pago firmada por Luis Guillermo de Portugal (La Haya, 7 de enero de 1647) «pour payer 700 florins au Peinctre Caudenberg", ANTT, ADAD, Caixa 5, Maço 3, Doc. 380.

44. Entre corchetes cuadrados remitimos al texto del catálogo publicado infra.

45. Responderían a asientos de los inventarios publicados por Dogaer, 1971. Codazzi, «Twee stucken perspective met figurkens van Biviano kosten beyde guld 40" (Dogaer, 1971: n. ${ }^{\circ} 48$ ); Foucquiéres, «Een curieus lantschapken van Focquier de figurkens van den fluweelen Bruegel heel got guld 200» (Dogaer, 1971: n. ${ }^{\circ}$ 160); Caravaggio «Een stucxken van druyven en vruchten van Michael Angelo Caravaggio oft Cavalier Mahot guld. 40» (Dogaer, 1971: n. $\left.{ }^{\circ} 47\right)$.

46. En Wilton House Guide (1908) se señala que se encuentra dispuesto "In the little ante room. Ceiling" (23).

IMAGO, NÚM. 9, 2017, 39-54 
Más afortunados en pinturas ${ }^{47}$ que en fama y reconocimiento, el recuerdo de los Príncipes de Portugal se vincula, así, a una impresionante colección de cuadros que una humilde recetería de papel nos permite conocer porque "l'on fait sçavoir que l'on vendra».

L'on faict scavoir; Que l'on vendra publicquement au plus haut offrant les Pintures cy suivant, appertenant à la Maison Mortuaire de Feu le Seigneur Prince de Portugal, le 3. de Mars 1670, à la Maison de Monseigneur l'Internonce Apostolica, en la ville de Bruxelles.

A Bruxelles, Chez Gilles Stryckwant, Imprimeur ordinaire des Estats de Brabant, 1670.

[1] Saint François en extase avec un Ange du Chevalier Bayoni.

BAGLIONE, Giovanni

[2] Nostre Dame, le petit Jesus, S. Jean, \& S. Joseph de Raphaël Urbino.

RAFAEL. Raffaello Santi

[3] La Judith de Michel Angelo Bronzino. BRONZINO. Agnolo

[4] Un Paysage rochers \& Montagnes avec figures de Benedetto di Castiglione. CASTIGLIONE, Giovanni Benedetto

[5] Nostre Seigneur mort entre deux Anges d'Hannibal Caratz.

CARRACCI, Annibale

[6] S. François avec une Croix en main d’Hannibal Caratz.

CARRACCI, Annibale

[7] Sainte Barbe de Michel Angelo Caravaggio.

CARAVAGGIO. Michelangelo Merisi da

[8] Quatre Paysages de Paul Bril.

BRILL, Paul
[9] Deux paysages avec figures de Gaspar Pussin.

DUGHET, Gaspard. Gaspard Poussin

[10] Un pourtraict d'un garcon avec une fleur de Caravaggio.

CARAVAGGIO. Michelangelo Merisi da

[11] Un Pot d'argent avec une couppe de Guillaume van Ast.

AELST, Willem van.

[12] Quatre Pots de fleurs de Mario. MARIO Nuzzi

[13] La decolation de S. Jean de Scipione Caetano.

PULZONE, Scipione, il Gaetano.

[14] Une Femme nuë dans un paysage de Joos van Winghe.

WINGHE, Joos van.

[15] Nostre Dame, Sainte Anne \& S. Joachim de Paulo Veronese.

VERONÉS. Paolo Caliari

[16] Beatus Paschalis de Pietro di Cortona. CORTONA. Pietro Berretini da

47. Su seguimiento e identificación de las pinturas se posterga por necesidad para otra ocasión, aunque merece la pena señalar que algunas entradas son altamente sugerentes, como "Le pourtraict d'un Cardenal de Velasquez", "Le temps de Van Dyck» o, entre otras muchas, «Un combat de deux femmes de Spagnolet» o los caravaggios. 
[17] Une conversation de Jean Bot. BOTH, Jan.

[18] Un pourtraict de Pordenone. PORDENONE. Giovanni Antonio de Sacchis.

[19] Un paysage avec figures de Foucquier. FOUQUIÈRES, Jacques

[20] Un paysage à la façon de Titien de Gaspar Pussin.

DUGHET, Gaspard. Gaspard Poussin.

[21] S. Pierre avec des mains de Jean Livens. LIEVENS, Jan.

[22] Deux blancs \& noirs du Polidore. POLIDORO CALDARA DA CARAVAGGIO.

[23] Un pot de fleurs sur planche de Mario. MARIO Nuzzi

[24] Mars \& Venus sur le lict de Carlo Venetiano.

SARACENI, Carlo.

[25] Une mande [i.e. manne] de fruicts de Caravaggio.

CARAVAGGIO. Michelangelo Merisi da.

[26] La Judith de Nicolas Pussin.

POUSSIN. Nicolas Poussin

[27] Un pourtraict d’un Religieux de Tintoreto.

TINTORETTO. Jacopo Robusti

[28] La resurrection de Lazare de Benedetti da Castiglione.

CASTIGLIONE, Giovanni Benedetto.

[29] Un tableau de fruicts $\&$ de fleurs de Bartholomeo Guido.
[30] Un pourtraict d'un Marechal de France de Porbus.

POURBUS, Frans

[31] Un pourtraict d'une femme avec un chien de Tintoretto.

TINTORETTO. Jacopo Robusti

[32] La predication de S. Jean d'Hannibal Caratz.

CARRACCI, Annibale

[33] S. Jean de Spagnolet. RIBERA El Españoleto. José de Ribera

[34] S. Jean de Correggio. CORREGGIO. Antonio Allegri da Correggio

[35] Deux larges Paysages de Titien. TIZIANO VECELLIO

[36] Un Ange de Leonardo da Vinci. LEONARDO DA VINCI

[37] S. André d'Andrea Sacchi. SACCHI, Andrea

[38] Une ruine en oval de Bot. BOTH, Jan.

[39] Un Berger de Giorgione. GIORGIONE. Giorgio da Castelfranco

[40] Le petit Bamboccio.

BAMBOCCIO. Pieter van LAER

[41] Une teste sur carton du Parmesan. PARMIGIANINO. Francesco Mazzola

[42] Un paysage avec figures de Francisco Molle.

MOLA, Pier Francesco? Franz van der Meulen 
[43] Un pourtraict d'un garçon de Gioseppino.

CAVALIER D’ARPINO. Giuseppe Cesari,

Il Giuseppino

[44] Un deluge blanc \& noir de Goltius. GOLTZIUS, Hendrick

[45] L'adoration des Roys de Paolo Veronese. VERONÉS. Paolo Caliari

[46] Ecce Homo du divin Morales. MORALES, Luis de, el Divino

[47] L’Ange qui éveille les Bergers de Bamboccio.

BAMBOCCIO. Pieter van LAER

[48] Deux mandes [i.e. mannes] de fleurs de Bodeson

BAUDESSON, Nicolas

[49] La presentation au Temple de nostre Seigneur de Jean Bellino.

BELLINI, Giovanni.

[50] Un paysage avec une femme qui marche de Francisco Molle.

MOLA, Pier Francesco?

Franz van der Meulen

[51] Une teste armée de Van Dyck apres Giorgone.

DYCK, Antoon van

[52] S. François de Paula sur papier.

[53] La teste de S. Pierre sans mains de Jean Lievens.

LIEVENS, Jan.

[54] La Cena Domini de Dominico Greco. EL GRECO, Domenico Theotocopuli

[55] Un paysage avec figures $\delta$ mouttons de Pietro Orrente ORRENTE, Pedro
[56] Un petit paysage de Foucquier. FOUQUIÈRES, Jacques

[57] Nostre Dame, le petit Jesus, S. Jean \& S. Joseph de Guido Reni.

RENI. Guido Reni

[58] Venus avec un Satyre du Vieux Dossa de Ferrare.

DOSSI. Giovanni Luteri. Dosso Dossi.

[59] Un petit pourtraict d'un garçon de Guido Reni.

RENI. Guido Reni

[60] Un petit pourtraict de Thomas Morus.

[61] Nostre Dame, le petit Jesus, S. Joseph, $\&$ S. Jerosme de Giorgione.

GIORGIONE. Giorgio da Castelfranco

[62] S. Jean de Parmesan. PARMIGIANINO. Francesco Mazzola

[63] La Lucrece de Monsieur de Wet. WET, Jacob de.

[64] Une marine de Paulo Bril. BRILL, Paul

[65] Un naufrage de Galeres de Filippo Napolitano.

FILIPPO NAPOLETANO. Filippo d'Angeli

[66] Deux perspectives du Vieux Viviano de Gennes.

CODAZZI, Viviano

[67] La Cena Domini de Tintoreto. TINTORETTO. Jacopo Robusti

[68] La naissance de Venus de Laurençio Bonomi. SABATTINI, Lorenzo. Laurentius Bononiensis 
[69] Le pourtraict d’un Cardenal de Velasquez. VELÁZQUEZ. Diego Rodríguez de Silva.

[70] Le temps de Van Dyck. DYCK, Antoon van

[71] Le festin des Dieux de Goltius. GOLTZIUS, Hendrick.

[72] Sainte Catherine de Guarçino d'Azento. GUERCINO. Giovanni Francesco Barbieri

[73] Venus \& Adonis de Lucas Cangiaccio. CAMBIASO, Luca.

[74] Nostre Seigneur au Jardin du Vieux Giacomo Bassan.

BASSANO, Jacopo. Jacopo da Ponte.

[75] Venus \& Adonis de Titien. TIZIANO VECELLIO

[76] Une piece de Feyt, \& la figure de Langhe Jan.

FYT, Jan, y Johann BOECKHORST, Lange Jan

[77] Nostre Dame, le petit Jesus, S. Joseph, $S$. François \& sainte Catherine, de Palme Giovene.

PALMA GIOVANE. Jacopo Negretti.

[78] S. Christophre d'Adam Elshamer. ELSHEIMER, Adam

[79] La tentation de S. Anthoine de Albordure.

DURERO. Albrecht DURER

[80] S. Sebastian de Nicolas Pussin. POUSSIN. Nicolas Poussin

[81] Deux bataille de Tempesta. TEMPESTA, Antonio
[82] Une teste d’un Cardenal de Titien. TIZIANO VECELLIO

[83] Le martire de sainte Ursule de Filippo Napolitano.

FILIPPO NAPOLETANO. Filippo d'Angeli

[84] La predication de saint Jean de Filippo Napolitano.

FILIPPO NAPOLETANO. Filippo d’Angeli

[85] Deux pieces de poissons del Gobbo di Napoli.

BONZI, Pietro Paolo, Gobbo de'Carracci

[86] Un paysage ou rocher de Nicolas Pussin. POUSSIN. Nicolas Poussin.

[87] Huict paysages du Chevalier Franquesano $\&$ autres Maistres. FRACANZANO

[88] L'embrassement de Troye de Filippo Napolitano FILIPPO NAPOLETANO. Filippo d'Angeli

[89] S. Anthoine de Padua de Carlo Napolitano.

VIVA, Carlo, Carlo Napolitano

[90] Le Limbe de Sotte Cleve. CLEVE, Cornelis van, Sotte Cleef

[91] Un pourtraict sur planche de Golbeen. HOLBEIN, Hans

[92] La teste d'un enfant de Carlo Loreneze. MELLIN, Charles. Carlo Lorenese

[93] Un pourtraict de Van Dyck. DYCK, Antoon van

[94] Un paysage \& ruïnes de Salvador Rosa. ROSA, Salvatore 
[95] Un combat de deux femmes de Spagnolet.

RIBERA El Españoleto. José de Ribera

[96] Un pourtraict de Spagnolet. RIBERA El Españoleto. José de Ribera

[97] Venus avec des Satires \& un Cupido de Martines.
[98] Un tableau representat S. Dominique Suriano du Chevalier Jean Lanfranco. LANFRANCO, Giovanni

[99] La petitte Magdaleine de Tintoretto. TINTORETTO. Jacopo Robust

\section{BIBLIOGRAFÍA}

Bayer, T. M. y Page, J. R. [2011]. The Development of the Art Market in England: Money as Muse, 1730-1900, Londres, Pickering \& Chatto.

Becmann, J. C. [1710]. Historie des Fürstenthums Anhalt [...] Fünfter, sechster und siebender Theil, [Zerbst, Gottfried Zimmermann].

BouzA, F. [2015]. "Don Antonio Prior do Crato y el horizonte portugués de la Leyenda Negra», en Y. Rodríguez, A. Sánchez y H. den Boer (eds.), España ante sus críticos. Las claves de la Leyenda Negra, Madrid, Iberoamericana-Vervuet, 117-138.

Briels, J, [1980]. "Amator Pictoriae Artis: De Antwerpse kunstverzamelaar Peeter Stevens (1590-1668) en zijn Constkamer», Jaarboek van het Koninklijk Museum voor Schone Kunsten Antwerpen, 137-266.

Brotton, J. [2006]. The Sale of the late King's Goods. Charles I and his art collection, Londres, Macmillan.

Castries, H. M. de [1906]. Les sources inédites de l'Histoire du Maroc de 1530 à 1845. Première Série. Dynastie Saadienne, 1530-1660, I, París, Ernest Leroux.

Clavería, C. [2017]. ¡Cuánto cuesta leer! Reflexiones sobre el precio de algunos libros españoles (1543-1806), Zaragoza, Prensas de la Universidad de Zaragoza.

DenucÉ, J. [1932]. De Antwerpsche Konstkamers inventarissen van kunstverzamelingen te Antwerpen in de $16^{e}$ en $17^{e}$ eeuwen. Amberes, De Sikkel.

- [1949]. Na Peter Pauwel Rubens: Documenten uit den Kunsthandel te Antwerpen in de XVIIe Eeuw van Matthijs Musson, Amberes, De Sikkel.

Deonna, W. [1929], Pierres sculptées de la vieille Genève (Collections lapidaires du Musée et documents hors du Musée), Ginebra, A. Kundig.

Dogaer, G. [1971]. "De inventaris der schilderijen van Diego Duarte», Jaarboek van het Koninklijk Museum voor Schone Kunsten Antwerpen, 195-221.

Dubert, I. [2002]. «Don Antonio, realidad y mito. El Prior do Crato, de la pretensión al trono de los Avís a Les psaumes confessionales franceses», en C. Fernández Cortizo (ed.), Universitas. Homenaje a Antonio Eiras Roel, Santiago, Universidade de Santiago de Compostela, 133-153.

FARIA, A. P. de [1917]. Descendance de D. António, prieur do Crato, XVIII Roy de Portugal, Lausana, Imp. Réunies. 
FernÁNDEZ-SAntos, J. [2014]. Juan Caramuel y la probable arquitectura, Madrid, CEEH.

GalesLoot, L. [1868]. "Vente à Londres de tableaux de David Teniers. Vente de la collection du Prince Ferdinand Alexandre de Portugal. Deux portraits peints par Antoine van Dyck. (xvII siècle). Notice», Annales de l'Académie Royale d'Archéologie de Belgique, 24, $2^{\mathrm{a}}$ serie, 4, 405-421.

GonZÁlez SARASA, S. [2016]. «Las menudencias impresas en los archivos y bibliotecas: clasificación, terminología y guía para su identificación», Cuadernos de Historia Moderna, 41/1, 169-198.

GutiéRrez, A. [1977]. La France et les français dans la litterature espagnole (1598-1665). Un aspect de la xenophobie en Espagne, Saint Etienne, Publications de l'Université.

Hantzsch, V. [1902]. "Beiträge zur älteren Geschichte der kurfürstlichen Kunstkammern in Dresden», Neues Archive für Sãchsische Geschichte und Altertumskunde, 23, 220-296.

Hermann, J. [2010]. «Um rei indesejado. Notas sobre a trajetória política de D. António, Prior do Crato", Revista Brasileira de História, 30, 59, 141-166.

Infantes, V. [2006]. Del libro áureo, Madrid, Calambur.

InfANTES, V. y MARTínez, A. [2016]. «Los catálogos del mercader de libros Gabriel de León (y otros catálogos del siglo XVII)», en P. Rueda y L. Agustí (eds.), La publicidad del libro en el mundo hispánico (siglos XVII-XX). Los catálogos de venta de libreros y editores, Madrid, Calambur, 17-42.

KAMP, J. L. J. vAN DE [1980]. Emanuel van Portugal en Emilia van Nassau, Assen, Van Gorcum, 1980.

Kennedy, J. [1764]. A New Description of the Pictures, Statues, Bustos, Basso-Relievos and Other curiosities in the Earl of Pembroke's House at Wilton, Londres, R. Baldwin, etc.

Lawler, J. [1898]. Book Auctions in England in the Seventeenth Century (1676-1700), Londres, Elliot Stock.

McElligott, J. [2005]. "Roger Morrice and the Reputation of Eikon Basilike in the 1680s", The Library, $7^{\text {th }}$ series, 6-2. 119-132.

Meulenbroek, B. L. (ed.) [1973]. Briefwisseling van Hugo Grotius, 9, La Haya, Martinus Nijhoff. Montias, J. M. [1989]. Vermeer and his Milieu. A Web of social History, Princeton, Princeton UP. - [2002]. Art at Auction in $17^{\text {th }}$ century Amsterdam, Amsterdam, Amsterdam UP.

PAePe, T. DE [2010]. «Diego Duarte II (1612-1691): A Converso's Experience in Seventeenthcentury Antwerp», Jewish History, 24-2, 169-193.

Paepe, T. DE [2016]. "Networking in high Society : the Duarte Family in seventeenthcentury Antwerp", Amberes, DIVA, <https://repository.uantwerpen.be/docman/irua/ b70c3e/138654.pdf> 29-06-17.

Piot, C. [1877]. Don Emmanuel, prétendant à la couronne de Portugal, et la famille de ce prince. Documents et particularités, Bruselas, F. Hayez.

Rueda, P. y Agustí. L. (eds.) [2016]. La publicidad del libro en el mundo hispánico (siglos XVII-XX). Los catálogos de venta de libreros y editores, Madrid, Calambur.

SAmuel, E. R. [1976]. "The disposal of Diego Duarte's stock of paintings 1692-1697», Jaarboek van het Koninklijk Museum voor Schone Kunsten Antwerpen, 305-324.

Serrão, J. V. [1956]. O reinado de D. António I, Prior do Crato. Vol. 1. 1580-1582, Coímbra, Instituto de Alta Cultura.

SousA, A. C. DE [1737]. Historia genealógica da Casa real portuguesa, III, Lisboa occidental, Joseph António da Sylva.

Speth-Holterhoff, S. [1957]. Les peintres flamands de cabinets d'amateur au XVII siècle, Bruselas, Elsevier. 
WenIGer, M. [2007]. «Das Sammeln spanischer Gemälde in Deutschland: Ein chronologischer Abriss», en K. Hellwig (ed.), Spanien und Deutschland: Kulturtransfer im 19.Jahrhundert = España y Alemania: Intercambio cultural en el siglo XIX, Madrid-Frankfurt am Main, Vervuet-Iberoamericana, 269-284.

Wiegers, G. [1996]. «Learned Moriscos and Arabic Studies in the Netherlands, 1609-1624», en J. LuedtKe (dir.), Romania Arabica. Fetschrift für Reinhold Kontzi zum, 70. Geburstag, Tubinga, Gunter Narr Verlag, 405-417.

Willems, J. [1935]. Le livre, l'estampe, l'édition en Brabant du XV' au XIX siècle, Gembloux, Duculot.

Wilton House Guide [1908], Londres, The Chiswick Press. 